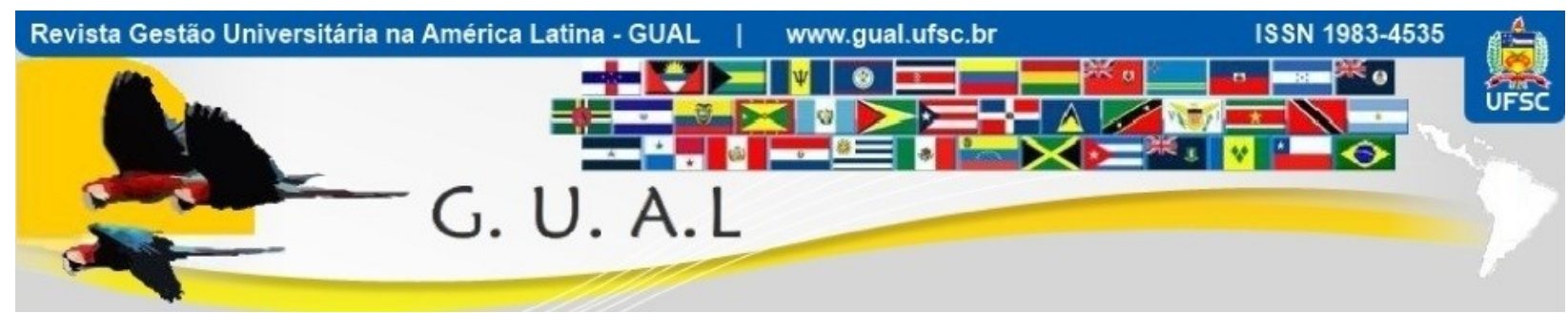

DOI: http://dx.doi.org/10.5007/1983-4535.2015v8n4p259

\title{
MAPEAMENTO CONCEITUAL DA INTER-RELAÇÃO ENTRE GESTÃO DO CONHECIMENTO, CAPITAL INTELECTUAL E MÉTRICAS DE QUALIDADE NAS UNIVERSIDADES
}

\section{MAPPING CONCEPT OF THE INTER- RELATIONSHIP BETWEEN KNOWLEDGE MANAGEMENT, INTELLECTUAL CAPITAL AND QUALITY METRICS IN UNIVERSITIES}

Neimar Sousa Pinto Pereira, Doutoranda Universidade do Minho (Portugal) neimar.anjo@gmail.com

Cleber Augusto Pereira, Mestre Universidade Federal do Maranhão kcleber@gmail.com

Telma Maria Chaves Ferreira da Silva, Doutoranda Universidade de Aveiro (Portugal) telmachaves@ua.pt

José Maria Paixão Filho, Mestre Universidade Federal do Maranhão (In memoriam)

Renato Pereira Monteiro, Doutorando Universidade de Aveiro (Portugal) renato.monteiro@poa.ifrs.edu.br

Edson José Borges, Doutorando Universidade de Aveiro (Portugal) edson.jose.borges@ua.pt

Recebido em 05/dezembro/2014

Aprovado em 05/abril/2015

Sistema de Avaliação: Double Blind Review 


\title{
RESUMO
}

O novo contexto das exigências imputadas às Instituições de Ensino Superior (IES) repercute em diversas dimensões dentro destas organizações. Neste contexto este trabalho apresenta um mapeamento conceitual resultante de uma revisão sistemática de literatura sobre a aplicação da Gestão do Conhecimento (GC), do Capital Intelectual (CI) e dos Modelos ou Métricas de Qualidade (MQ) aplicados às universidades. Pretendeu-se compreender e identificar como as técnicas de GC, as aplicações do CI e a utilização ou adoção de MQ podem funcionar como instrumentos de apoio diferenciais as IES. A revisão de literatura baseou-se em artigos publicados em veículos de comunicação de reconhecida qualidade e com fator de impacto relevante. A contribuição principal deste trabalho residiu na elaboração de proposta de mapeamento integrativo dos conceitos, utilizando-se um mapa conceitual construído colaborativamente entre os autores, e, na elaboração do modelo conceitual do trabalho derivado deste mapeamento. Estes modelos permitiram identificar, de forma visual, a ocorrência de inter-relacionamentos entre os conceitos, que não seriam de fácil evidenciação num contexto meramente textual.

Palavras-chave: Gestão do conhecimento. Capital intelectual. Métricas de qualidade. Universidades. Mapa conceitual.

\begin{abstract}
The new context of the requirements allocated to higher education institutions (HEI) resonates in various dimensions within these organizations. Therefore, this work presents a conceptual resulting after a systematic review mapping about literature application at Knowledge Management (KM), the Intellectual Capital (IC) and Templates or Metric Quality (MQ) applied to the universities. It was intended to understand and identify how GC techniques, IC applications, and the use or adoption of MQ can function as tools to support differential of IES. The literature review was based on articles published in scientific journals of qualified with relevant impact factor. The main contribution of this paper is focused on the preparation of draft integrative mapping of concepts, using a concept map built collaboratively among authors, and in preparation of the conceptual work model mapping derivative as thereof. These models indicate, visually, an occurrence of inter-relationship between concepts which would not be easy disclosure a purely textual context.
\end{abstract}

Keywords: Knowledge Management. Intellectual Capital. Quality Metrics. Universities. Conceptual Map. 


\section{INTRODUÇÃO}

As novas aplicações em informática e o contexto de globalização do ensino superior têm interferido positivamente na forma clássica de ensinar e no ambiente das universidades, colaborando para uma possível ruptura da tradicional estrutura e gestão do ensino superior. As relações de trabalho, ante aos modernos modelos tecnológicos de produção flexível, tiveram de se adequar à luz dos conceitos de produtividade e estratégias operacionais. Nesse cenário, o contexto da educação superior, que tem como foco a aprendizagem e a disseminação do conhecimento, viu-se forçada a dar respostas rápidas às demandas impostas pela nova ordem mundial.

Neste contexto, emerge a necessidade de adequação das universidades através da criação de propostas de certa forma inovadoras que visem resgatar o espírito científico, elevar a qualidade da educação com a introdução de metodologias interdisciplinares, agregando valor qualitativo aos cursos e às disciplinas, desenvolvendo projetos que privilegiem o valor da descoberta associado à cultura regional, do desenvolvimento da investigação permitindo o questionamento, como uma visão crítica do mundo contemporâneo.

Com base neste cenário, este trabalho realiza uma revisão sistemática de literatura com o objetivo de permitir a compreensão e identificação de como podem ser aplicadas as técnicas de Gestão do Conhecimento, a utilização de Métricas de Qualidade e a Gestão do Capital Intelectual nas IES.

Os objetivos específicos consistem na avaliação da literatura das diferentes abordagens que permitem mensurar a qualidade, dentro do contexto da GC e do CI, aplicáveis ao ensino superior; identificar os principais mecanismos de garantia da qualidade em prática pelas IES; avaliar a ocorrência da inter-relação entre estes elementos, demonstrando-a de forma gráfica.

\subsection{METODOLOGIA}

A seção a seguir descreve a metodologia utilizada para a revisão sistemática de literatura, detalhando a forma de pesquisa e seleção dos artigos em journals e periódicos. $\mathrm{Na}$ seção 1.2.1 são descritos os procedimentos de investigação realizados nas bases de dados referenciais e as principais bases utilizadas. Na seção 1.2.2 é apresentado o tipo de pesquisa e sua caracterização e a forma utilizada para a visualização da pesquisa. 


\section{MAPEAMENTO CONCEITUAL DA INTER-RELAÇÃO ENTRE GESTÃO DO CONHECIMENTO, CAPITAL INTELECTUAL E MÉTRICAS DE QUALIDADE NAS UNIVERSIDADES \\ DOI: http://dx.doi.org/10.5007/1983-4535.2015v8n4p259}

\subsubsection{Revisão Sistemática de Literatura}

Para a revisão sistemática de literatura adotou-se investigação bibliográfica em veículos de publicação: artigos e journals com Fator de Impacto (FI) relevante. Foram priorizadas as publicações recentes, sendo consideradas as dos últimos oito anos, de 2006 a 2013, consideraram-se ainda algumas publicações clássicas e reconhecidas independente de sua data de publicação.

Foi realizada pesquisa no Brasilian Journal of Information Science avaliando-se todas as publicações do período de 2006 (inclusive) até 2013. Nos critérios de busca utilizaram-se das palavras-chave "gestão do conhecimento", "capital intelectual”, "intangíveis", "métricas de qualidade" e "universidade", sendo previamente selecionados trinta e dois artigos. Após a leitura e revisão dos artigos foram selecionados nove artigos deste journal.

No periódico Journal of Intellectual Capital Content a pesquisa considerou somente os artigos que apresentavam a palavra-chave "university" em qualquer campo e conjuntamente com os termos "intellectual capital" e "intangible", aceitando-se ainda com o operador "or" (exclusive) as palavras-chave "quality metrics". Dos resultados previamente selecionados, após a aplicação de filtros de conteúdo e após a revisão sistemática dos resumos e/ou abstracts utilizados vinte e seis artigos filtrados no período dos últimos oito anos. Os demais artigos utilizados neste trabalho foram selecionados com base na leitura das referências citadas pelos autores dos artigos principais.

Da amostra acima referenciada, os resultados foram exportados para o gerenciador de referências e seus documentos completos devidamente anexados. Para esta tarefa e controle das citações utilizou-se o software Mendeley Desktop, específico para este fim.

O Mendeley permite sistematizar a forma como o material coletado é utilizado e administrar com praticidade as informações de autoria dos artigos selecionados para o referencial teórico. Além do gerenciamento, essa ferramenta torna possível ler e compartilhar artigos, fazer marcações em seu conteúdo, também possibilitando realizar tais atividades online (MENDELEY, 2013).

\subsubsection{Metodologia da Investigação, Caracterização e Apresentação da Pesquisa}

A pesquisa é caracterizada como bibliográfica realizada por meio de journals e outros trabalhos acadêmicos, concordando com Silva (2008, p. 54) que explica que uma pesquisa bibliográfica fornece dados para qualquer tipo de pesquisa e é feita com base em referências teóricas publicadas em livros, revistas, periódicos ou artigos científicos. 


\section{MAPEAMENTO CONCEITUAL DA INTER-RELAÇÃO ENTRE GESTÃO DO CONHECIMENTO, CAPITAL \\ INTELECTUAL E MÉTRICAS DE QUALIDADE NAS UNIVERSIDADES \\ DOI: http://dx.doi.org/10.5007/1983-4535.2015v8n4p259}

Com base nos achados da revisão de literatura e após a delimitação do quadro teórico e análise das dimensões discutidas nos trabalhos selecionados, foi possível identificar nos estudos as principais aplicações realizadas nas universidades. A maneira escolhida para representar visualmente, na forma de proposições, as relações significativas entre os conceitos descobertos, foi a utilização de um diagrama hierárquico - o mapa conceitual, muito utilizado nos estudos de gestão do conhecimento.

O mapa conceitual pode ser entendido como um diagrama bidimensional que tem como principal função demonstrar conceitos hierarquicamente organizados e as relações entre conceitos. As linhas de conexão representam as relações entre conceitos. (MOON et al., 2011).

O software computacional utilizado para a elaboração do mapa conceitual foi o Cmap Tools Knowledge Modelling Kit ${ }^{1}$. Trata-se de uma ferramenta metodológica que utiliza a teoria de assimilação para determinar o que o estudante sabe ou descobriu durante a revisão de literatura. (CAÑAS et al., 2000, p. 1-2)

O recente estudo de Hagemans, Van Der Meij e De Jong (2013) relata a experiência de estudantes que visualizaram o mapa conceitual, e superaram os estudantes que realizaram apenas a análise descritiva. Os resultados mostraram que a utilização de mapas conceituais contribui com a melhoria da aprendizagem e descoberta de relações entre conceitos.

Desta forma, pelas contribuições da utilização de mapas conceituais acima descritas, justifica-se a aplicação de mapa conceitual para demonstrar os conceitos e relacionamentos descobertos.

Durante o desenvolvimento do mapa conceitual deste trabalho, os autores trabalharam colaborativamente na criação do mapa conceitual utilizando o software CMap Tools, a experiência de desenvolvimento colaborativo resultou em uma proposta de mapeamento integrativo bastante madura e sintetizada.

\section{GESTÃO DO CONHECIMENTO E O CAPITAL INTELECTUAL}

Esta seção aborda a revisão de literatura sobre a GC e o CI com foco de sua aplicação nas universidades. Foram ainda avaliadas a integração destes com o sistema de métricas de qualidade citadas ou em uso nas universidades.

\footnotetext{
${ }^{1}$ Software livre desenvolvido pelo Institute of Human and Machine Cognition (IHMC).

Disponível em $<$ http://cmap.ihmc.us $>$
} 


\subsection{O CONHECIMENTO, A GESTÃO DO CONHECIMENTO E O CAPITAL} INTELECTUAL

Iniciando a definição de conhecimento é importante frisar que os termos: dado, informação e conhecimento não são sinônimos: Dados são quaisquer sinais, números, palavras, figuras, sons, textos, gráficos, fotos sem contexto, ou seja, são sinais desprovidos de interpretação ou significados (SCHREIBER, 2002). A Informação, por sua vez, constitui dados com algum contexto ou significado; isto é, são dados dotados de relevância com o objetivo de informar algo a alguém (TIWANA, 2002). Por conseguinte, Dean e Gottschalk (2007) referem-se a conhecimento como informação combinada com experiência, contexto, interpretação, reflexão e criatividade. E, para Davenport e Prusak (1998), o conhecimento tem origem e aplicação na mente de seus detentores.

O conhecimento é um ativo que começa por estar presente no indivíduo e que cresce com as interações do individual para o mercado, conforme a pode ser visualizado na Figura 1.

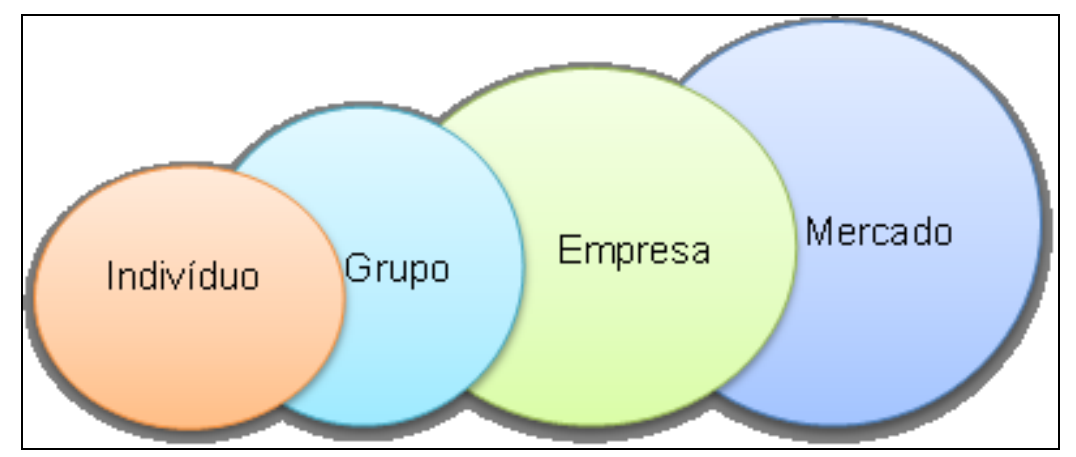

Figura 1 Dimensões do conhecimento organizacional

Fonte: adaptado de Amaral; Pedro (2004)

O conhecimento é distinto de duas formas, a primeira forma é conhecimento tácito que é suportado pelos indivíduos da organização, esta apoia as habilidades pessoais e específicas do individuo. A segunda forma, conhecimento explícito é o conhecimento codificado, formalmente sistematizado numa linguagem (NONAKA; TAKEUCHI, 1995).

Tabela 1 Conversão do conhecimento tácito para explícito

\begin{tabular}{|c|c|}
\multicolumn{1}{|c|}{$\begin{array}{c}\text { Conhecimento tácito } \\
\text { (subjetivo) }\end{array}$} & $\begin{array}{c}\text { Conhecimento Explicito } \\
\text { (Objetivo) }\end{array}$ \\
\hline $\begin{array}{c}\text { Conhecimento de experiência } \\
\text { (Corpo) }\end{array}$ & $\begin{array}{c}\text { Conhecimento de racionalidade } \\
\text { (Mente) }\end{array}$ \\
\hline $\begin{array}{c}\text { Conhecimento simultâneo } \\
\text { (aqui e agora) }\end{array}$ & $\begin{array}{c}\text { Conhecimento sequencial } \\
\text { (lá e então) }\end{array}$ \\
\hline $\begin{array}{c}\text { Conhecimento análogo } \\
\text { (Prático) }\end{array}$ & $\begin{array}{c}\text { Conhecimento digital } \\
\text { (Teoria) }\end{array}$ \\
\hline
\end{tabular}

Fonte: Adaptado de Nonaka; Takeuchi (1995, p.61) 


\section{MAPEAMENTO CONCEITUAL DA INTER-RELAÇÃO ENTRE GESTÃO DO CONHECIMENTO, CAPITAL INTELECTUAL E MÉTRICAS DE QUALIDADE NAS UNIVERSIDADES \\ DOI: http://dx.doi.org/10.5007/1983-4535.2015v8n4p259}

A representação de Nonaka e Takeuchi (1995, p.60-67), constante na Tabela 1, permite a conversão da tipagem do conhecimento, de tácito para explícito, no sentido de aproximação de sua representação objetiva.

Kelly (2004) destaca que o conhecimento explícito deve ser integralmente descrito e armazenado de forma algorítmica transmissível; pois o conhecimento tácito é pessoal e individual de seu titular. Para a universidade, a conceituação de capital intelectual sustenta que quando há uma transferência de uma categoria para outra, um capital de conhecimento adicional é gerado para a instituição. Aplicar o conhecimento criado para o ensino em sala de aula, num processo de transferências explícito-tácito, é a chave para alcançar melhorias e valor agregado para a universidade. E o conhecimento para alcançar os efeitos da variação positiva no desempenho da organização deverá ser acumulado, ao longo do tempo, no reservatório da instituição, ou seja, um mecanismo através do qual a sistémica conhecimento prático é codificado e armazenado para uso futuro.

Em relação à gestão do conhecimento Lloria (2008) e Sellar e Lingard (2013) apontam que a GC implica em uma série de políticas e diretrizes que permitem a criação, divulgação e institucionalização do conhecimento, a fim de atingir os objetivos da empresa, e discute a história, evolução e desenvolvimento da Organização para a Cooperação Econômica e Desenvolvimento (OCDE), em vários segmentos da GC aplicados ao ensino, independente do nível educacional, avaliando o crescente impacto do seu trabalho educativo.

Sousa (2010) complementa que a GC pode envolver qualquer atividade relacionada à captura, uso, e, compartilhamento de conhecimento pelas organizações de qualquer tipo.

Quanto ao capital intelectual, foi realizada uma recente pesquisa do estado da arte por Labra e Sanches (2013, p. 582-607) com o objetivo de revisar a literatura sobre o capital intelectual na Espanha para identificar e comparar os principais modelos para medir e relatar CI ao nível do país. A pesquisa abrangeu o período de janeiro de 2000 a dezembro de 2012. Os modelos utilizados para medir intangíveis foram analisados e comparados e os achados indicaram que tal literatura se apresenta em crescente desenvolvimento.

Um fato histórico relevante ao capital intelectual ocorreu em maio de 1995, na publicação do primeiro relatório anual de capital intelectual, realizado pela organização de serviços financeiros e seguros Skandia. A organização havia investigado o capital intelectual durante 4 anos sob a liderança de Leif Edvinsson. O método de capital intelectual da Skandia estava fundamentado na ideia de que, o verdadeiro valor de desempenho de uma organização 


\section{MAPEAMENTO CONCEITUAL DA INTER-RELAÇÃO ENTRE GESTÃO DO CONHECIMENTO, CAPITAL INTELECTUAL E MÉTRICAS DE QUALIDADE NAS UNIVERSIDADES \\ DOI: http://dx.doi.org/10.5007/1983-4535.2015v8n4p259}

está na capacidade de criar valor sustentável, focando a visão de negócios e a estratégia adequada (EDVINSSON; MALONE, 1997; SCHINDLER; JAITNER, 2003)

No Líbano, Safieddine, Jamali e Noureddine (2009) exploraram a relação entre o governo da organização e o capital intelectual num contexto acadêmico. Através de pesquisa empírica com questionário aplicado on line e enviado aos professores em tempo integral de todas as faculdades. As perguntas foram elaboradas com base na revisão de literatura, objetivando identificar quais são os fatores considerados importantes por membros do corpo docente para atrair o capital intelectual e a gestão eficiente da Universidade. Esta abordagem também permitiu identificar quais são as áreas fortes de governança da organização, bem como, as áreas nas quais melhorias deveriam ser introduzidas para atrair e reter o capital intelectual da universidade. Com base nas respostas conclui-se que a governança da organização e o capital intelectual são interligados. Definem ainda que o capital intelectual é a força latente que aumenta a capacidade de uma organização para atrair mais capital intelectual.

\subsection{A GESTÃO DO CONHECIMENTO NAS UNIVERSIDADES}

O trabalho de Bem e Coelho (2013) propôs uma revisão sistemática de literatura com a finalidade de identificar aplicações e tendências de gestão do conhecimento na forma de mapa conceitual na área de Ciência da Informação e ressalta que é necessário que a GC faça parte de suas rotinas, de modo a permitir que estas se beneficiem das melhorias que podem ser conseguidas por meio da GC, aprimorando os serviços e satisfazendo os usuários.

O artigo de Canterle e Favaretto (2008) apresenta um modelo referencial de gestão aplicado à qualidade na universidade, sintetizando uma descrição dos indicadores essenciais ao desenho de um modelo de gestão universitária mais próxima da realidade. O modelo traz a possibilidade de executar uma gestão ancorada em parâmetros reais mensurados por indicadores qualitativos e quantitativos, sem ser prescritivo.

A proposta de Carmo e Pecegueiro (2012, p. 97-109) utiliza técnicas de GC para composição e disponibilização dos documentos científicos produzidos em um Repositório Institucional. Ressalta a importância do documento como suporte e representação da informação e a praticidade do formato digital para a disponibilização da produção científica institucional à comunidade.

$\mathrm{Na}$ mesma direção do trabalho apresentado acima, porém num cenário em que a universidade utiliza diferentes sistemas de informação, o trabalho de Ciupak, Boscarioli e 


\section{MAPEAMENTO CONCEITUAL DA INTER-RELAÇÃO ENTRE GESTÃO DO CONHECIMENTO, CAPITAL INTELECTUAL E MÉTRICAS DE QUALIDADE NAS UNIVERSIDADES \\ DOI: http://dx.doi.org/10.5007/1983-4535.2015v8n4p259}

Catarino (2013) faz uso de tecnologias de Business Inteligence (BI) para desenvolver um protótipo para a recuperação e integração das informações oriundas de sistemas diversos.

Preocupado com a GC na comunicação do meio científico, o artigo de Leite e De Souza Costa (2007) propôs-se a investigar a relação, em nível conceitual, entre a gestão do conhecimento e os processos de comunicação científica, tendo em vista as peculiaridades do contexto e do conhecimento científico, propondo um modelo conceitual de gestão do conhecimento científico no contexto acadêmico. Esta abordagem é interessante no contexto universitário considerando-se que, durante a totalidade do ciclo do conhecimento, nota-se que o pesquisador utiliza inúmeros canais de comunicação.

Migrando os dois tópicos aqui discutidos: GC e CI, identificou-se o artigo de Li, Pike e Haniffa (2008) que investiga a relação entre a divulgação do capital intelectual e as variáveis de governança corporativa em uma amostra de 100 empresas do Reino Unido. Os resultados da análise indicaram associação significativa e influência da divulgação do CI com todos os fatores de governança.

Do ponto de vista da análise da divulgação do CI nas universidades, o trabalho de Bezhani (2010) realiza uma investigação, sem estudos precedentes no Reino Unido, com o objetivo de analisar a quantidade, a natureza e a divulgação voluntária de CI nos relatórios anuais em 30 das 118 universidades ranqueadas como melhores, identificando como principal achado a baixa quantidade divulgada de CI.

Para identificar a relevância atribuída à publicação de informações sobre capital intelectual nas universidades, Córcoles; Peñalver e Ponce (2011) analisaram as universidades públicas da Espanha para atingir dois objetivos: O primeiro foi identificar a importância atribuída pelos stakeholders, à publicação de informações sobre capital intelectual. Os stakeholders identificados consideram essencial que as universidades forneçam informações sobre o capital intelectual, a fim de fazer o modelo de informação da contabilidade mais relevante. Como segundo objetivo, procuraram identificar a extensão da procura dos diferentes usuários para obter informações sobre capital intelectual. Os resultados mostraram uma procura específica de capital intelectual para os diferentes utilizadores e serviram como base para ratificar a necessidade de incluir esta informação no modelo contabilístico na forma de um relatório de capital intelectual.

Sobre esta inter-relação entre a as práticas de gestão do conhecimento e o relato do capital intelectual, inúmeros estudos foram identificados nesta investigação no período entre 1998 e 2007 e constituem-se como iniciativas para promover sua associação e necessidade de 
inter-relacionamento na nova economia (BONTIS et al., 1999; BUENO; SALMADOR; RODRÍGUEZ, 2004; CERBIONI; PARBONETTI, 2007; DE PABLOS, 2002; FIRER; WILLIAMS, 2003; GUTHRIE; PETTY; JOHANSON, 2001; KEENAN; AGGESTAM, 2001; MOURITSEN, 1998; RIAHI-BELKAOUI, 2003; TEECE, 2003; TSENG; JAMES GOO, 2005).

Quanto aos estudos realizados em universidades em inúmeros países, a revisão de literatura demonstrou que em sua maioria, os achados apresentam a necessidade de evolução e maturidade destas para o adequado relato do uso do CI ao público alvo (ABEYSEKERA; GUTHRIE, 2005; BRENNAN; SHAH, 2000; DE PABLOS, 2002; GEUNA; NESTA, 2006; KAMLA; GALLHOFER; HASLAM, 2012; LEITNER, 2004; O’SHEA et al., 2005; PETTY; GUTHRIE, 2000; SÁNCHEZ; ELENA, 2006).

\subsection{UMA DISCUSSÃO SOBRE OS MODELOS DE QUALIDADE NO ENSINO SUPERIOR}

No ambiente de gestão do ensino superior contemporâneo a realidade da busca por qualidade têm sido uma vertente cada vez mais relevante conforme Seymour (1992) afirma que a qualidade tem sido vista como algo que pode e deve ser gerida a melhorada.

No Brasil, houve um conjunto de políticas de educação superior implementadas a partir de 1995, surgindo a necessidade de criar instrumentos legais para sustentar e avaliar de forma eficaz a expansão quantitativa e diversificada da educação superior. A avaliação se tornou um relevante instrumento para informar acerca da qualidade e capacidade profissional oferecidas pelos cursos, do mesmo modo que identifica as instituições de ensino superior mais ajustadas as exigências da sociedade (DIAS SOBRINHO, 2010).

Nesta direção, Stensaker (2007) aponta que modelos de gestão como o Total Quality Management (TQM) primeiramente considerado no ensino superior, repercutiu em inúmeras discussões sobre seu potencial para a categoria e suas respectivas implicações institucionais e sociais para a educação.

Em outro estudo clássico, Seymour (1991) aponta alguns motivos que podem ter levado à falha da institucionalização da TQM nas IES como: resistência à mudanças, falta de compromisso administrativo, dificuldades oriundas à formação de pessoal, obstáculos para a aplicação das ferramentas de TQM nas IES, além de dificuldades de gerir um trabalho em equipe. 


\section{MAPEAMENTO CONCEITUAL DA INTER-RELAÇÃO ENTRE GESTÃO DO CONHECIMENTO, CAPITAL INTELECTUAL E MÉTRICAS DE QUALIDADE NAS UNIVERSIDADES \\ DOI: http://dx.doi.org/10.5007/1983-4535.2015v8n4p259}

Posteriormente à realidade exposta pela afirmativa de Stensaker (2007), e indo de encontro ao sugerido por Seymour (1991), uma pesquisa conduzida por Birnbaum e Deshotels (1999), aplicada em quatrocentas e sessenta e nove IES dos EUA conclui que a adoção do TQM no ensino superior poderia ser considerada como um mito e ilusão.

A ideia a seguir seria a possibilidade de adoção das normas ISO como forma de obter vantagens para as IES uma vez que a aplicação destas normas em uma organização tende a lhe conferir maior organização, produtividade e credibilidade.

Dentre estes modelos pode-se citar a norma ISO 9001:2000 que estabelecia o controle de processo acompanhado o antes e o durante o processo além de exigir o comprometimento da gestão para a integração da qualidade e introduzindo a visão de foco no cliente.

Outro modelo de excelência oriundo das indústrias, European Foundation for Quality Management (EFQM) foi aplicado às IES, preconizado como uma ferramenta de autoavaliação e de melhoria contínua com base na utilização eficiente dos meios, como prerrogativa de alcance de excelentes resultados e possibilitando às organizações a obtenção de um panorama abrangente da sua situação global em determinado momento. Este modelo propõe fornecer um feedback acerca da eficácia das abordagens adotadas em todas as suas atividades.

O modelo de Melhoria de Equilíbrio e Responsabilidade constitui-se em um tema central na qualidade conforme a proposta de Gosling e D’Andrea (2001). Em seu estudo, os autores argumentam que um sistema de qualidade deve ser não somente executar uma função reguladora, mas que apresente funções para melhorar a qualidade da experiência educacional (GOSLING; D’ANDREA, 2001, p. 11).

Os autores afirmam ainda que podem ocorrer conflitos derivados das diferenças de valores inerentes aos processos de regulação e melhoria da qualidade, tendo em vista que a garantia da qualidade concentra-se na avaliação da qualidade e desenvolvimento educacional concentra-se na melhoria da qualidade (GOSLING; D'ANDREA, 2001, p. 11-12). Desta forma, ressaltam a importância do desenvolvimento integral de um modelo de ensino que consiga combinar a valorização da aprendizagem e do ensino com a qualidade e padrões de monitoramento de processos em uma instituição de ensino superior.

Como resultados deste modelo apontam-se algumas vantagens para a IES, funcionários e alunos do ponto de vista: do estabelecimento de vínculo entre a segurança da qualidade e o desenvolvimento educacional; de facilitar o diálogo entre os responsáveis pela garantia de qualidade e os responsáveis para o desenvolvimento educacional sobre as políticas 


\section{MAPEAMENTO CONCEITUAL DA INTER-RELAÇÃO ENTRE GESTÃO DO CONHECIMENTO, CAPITAL INTELECTUAL E MÉTRICAS DE QUALIDADE NAS UNIVERSIDADES \\ DOI: http://dx.doi.org/10.5007/1983-4535.2015v8n4p259}

de qualidade internas e externas de avaliação e procedimentos; da cooperação entre os processos de garantia de qualidade e os processos de melhoria de qualidade, culminando em uma divulgação mais eficaz das políticas educacionais dentro de uma instituição e manutenção de bons padrões gerais de oferta institucional. (GOSLING; D’ANDREA, 2001, p. 12).

Um ponto crítico exposto pelo modelo é a falta de diálogo entre os responsáveis pelo desenvolvimento curricular e a unidade de apoio à aprendizagem dos alunos, estas que, na maioria dos casos, apresentam também responsabilidades em áreas separadas. Esta integração, conforme Harvey (1998) é indispensável para a garantia da qualidade de ambas.

Inúmeros modelos populares e oriundos da indústria foram aplicados às IES na busca de resultados significantes, embora Harvey (1995) afirme que a abordagem mais recente deve dedicar-se ao desenvolvimento de modelos de gestão da qualidade com foco nas características específicas das IES.

Para Csizmadia, Enders e Westerheijden (2008, p. 24) a gestão da qualidade pode ser definida como um termo mais abrangente que engloba políticas, conceitos, abordagens, ideias, sistemas e processos destinados a assegurar a manutenção sistemática e reforço da qualidade em uma IES. Trow (1994) destaca que a gestão da qualidade pode estar orientada a diversos fins como a melhoria de qualidade ou para tornar as IES mais responsáveis, eficientes e com transparência.

Brennan e Shah (2000, p. 5) afirmam que existe uma relação íntima de gestão da qualidade com processos de decisão e ação. Diferenciam os valores de qualidade como sendo acadêmicos, gerenciais, pedagógicos e focados no emprego e prescrevem a necessidade de uma aderência destes valores quando da escolha da abordagem para a gestão para garantir o sucesso em sua implantação.

As dimensões acadêmica, gerencial, pedagógica e com foco no emprego (mercado) estabelecidas por Brennan e Shah (2000) são semelhantes às dimensões hoje utilizadas pelas agências de acreditação que avaliam: corpo docente, coordenador, projeto pedagógico e infraestrutura dos cursos.

$\mathrm{Na}$ dimensão "acadêmica" o foco relaciona-se com a autoridade do professor e considera relevantes os valores acadêmicos para a concepção de qualidade, sendo que o escopo para definição e avaliação da qualidade é limitado e variam de acordo com cada instituição (BRENNAN; SHAH, 2000, p. 14). Em uma análise desta dimensão nos tempos atuais a similaridade é muito grande, considerando-se que na dimensão de avaliação do corpo 


\section{MAPEAMENTO CONCEITUAL DA INTER-RELAÇÃO ENTRE GESTÃO DO CONHECIMENTO, CAPITAL INTELECTUAL E MÉTRICAS DE QUALIDADE NAS UNIVERSIDADES \\ DOI: http://dx.doi.org/10.5007/1983-4535.2015v8n4p259}

docente, durante os processos de acreditação, são consideradas as unidades curriculares ministradas pelos professores e sua aderência com a formação acadêmica destes profissionais. $\mathrm{Na}$ dimensão "gerencial”, de forma essencial, um indicador de qualidade do sistema de gestão é que deve ser descentralizado, com foco em características disciplinares e aplicação de padrões de qualidade diferentes. $O$ tipo de gestão apresenta políticas institucionais e procedimentos como foco principal da avaliação, de forma subjacente as boas práticas de gestão podem ser entendidas como o fator-chave de produção de qualidade. As características de qualidade são consideradas invariantes em toda a IES (BRENNAN; SHAH, 2000, p. 14 $15)$.

Analisando a dimensão gerencial, em relação à abordagem atual do coordenador do curso (gestor), identificam-se afinidades quando se tem o entendimento de que a gestão da IES em seus inúmeros cursos deve ser descentralizada, entendendo-se esta característica como preservatória das características peculiares de cada curso. Todavia em cada curso a gestão poderá ser centralizada no sentido de manter o acoplamento com as estratégias institucionais e utilização de padrões de qualidade mais coerentes com a sua área de atuação.

A dimensão "Pedagógica" centraliza-se nas pessoas e nos aspectos pedagógicos do processo, habilidades de ensino e métodos utilizados, formação de pessoal e desenvolvimento. Estes aspectos são considerados invariantes em toda a IES (BRENNAN; SHAH, 2000, p. 14-15). O que ocorre nesta dimensão reflete a realidade atual do cenário de ensino superior brasileiro, quando se caracteriza o "projeto pedagógico" como as diretrizes norteadoras dos padrões de ensino, pesquisa e extensão.

Finalmente, a dimensão foco no emprego centraliza-se nos fins da aprendizagem, resultados da aprendizagem, padrões e características de saída de graduados. Esta abordagem lida com os requisitos do mercado, onde os clientes podem ser vistos como os empregadores de licenciados. Para Brennan e Shah (2000, p. 15) esta dimensão tende a levar em consideração os objetivos específicos e as características fundamentais de uma educação de qualidade.

As categorias propostas oferecem uma forma simples e eficaz de identificar as principais características e o foco de um determinado modelo de gestão da qualidade, embora não sejam capazes de capturar a complexidade das diferentes abordagens para a gestão da qualidade. A comparação com as práticas atuais de avaliação e acreditação as têm validado.

A próxima seção apresentará a inter-relação dos conceitos trabalhados na revisão de literatura através do mapa conceitual. 


\subsection{MAPA CONCEITUAL DA REVISÃO DE LITERATURA}

Foi desenvolvido um diagrama bidimensional da revisão de literatura, o mapa conceitual que, conforme explicado na seção 1.2.2 metodologia da investigação, caracterização e apresentação da pesquisa, tem como principal função demonstrar, de forma hierarquicamente organizada, os conceitos-chave e as relações entre estes, relacionando-os com os respectivos autores.

O mapa conceitual, demonstrado na Figura 2, foi elaborado com base no quadro teórico apresentado nas seções anteriores. Utilizou-se do mapa conceitual para identificar, de forma mais clara e evidente, que a Gestão do Conhecimento, o Capital Intelectual e os Modelos e/ou Métricas de Qualidade encontram-se intimamente relacionados no ambiente do ensino superior das universidades.

A experiência compartilhada entre os autores durante a elaboração colaborativa do mapa conceitual pode ser avaliada como integrativa e produtiva, considerando-se o know-how diversificado entre as diversas áreas do saber individual.

Note-se que os conceitos-chave estão dispostos em negrito no mapa conceitual: modelos ou métricas de qualidade, gestão do conhecimento, capital intelectual e ensino superior, neste contexto representado pelas universidades. As relações entre os conceitoschave e os conceitos descobertos na revisão de literatura são descritos pelas ligações e sentido das setas, que representam as relações conceituais.

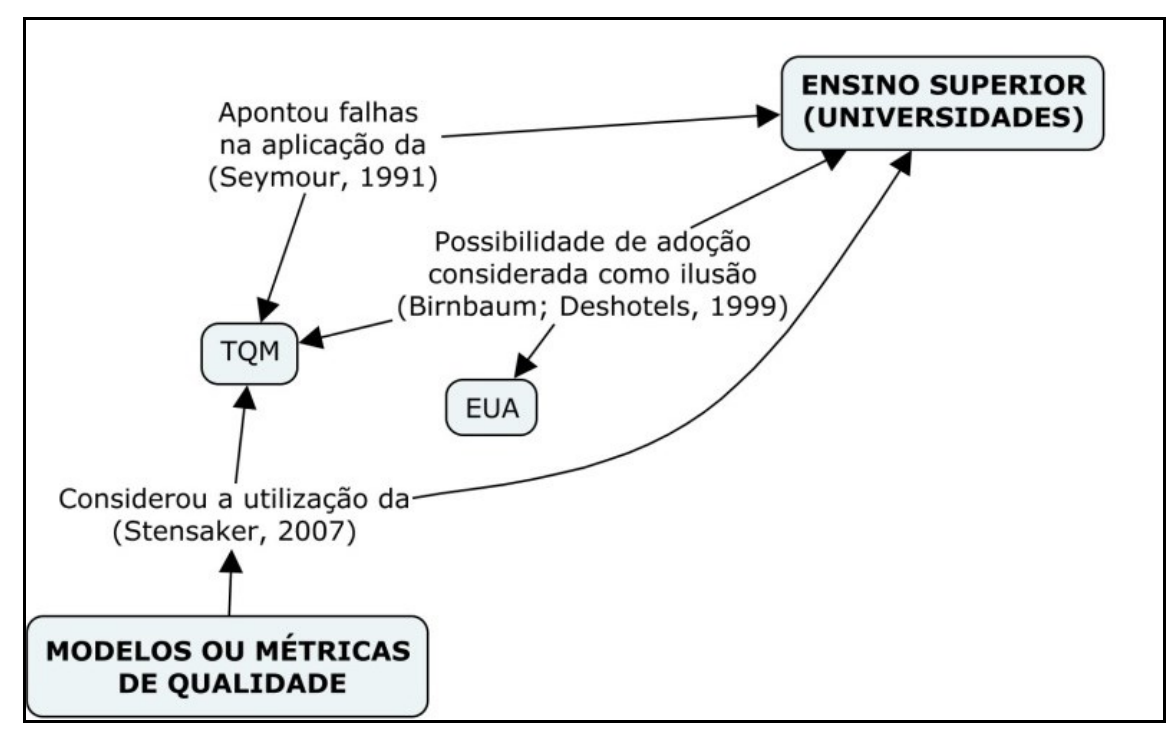

Figura 2 Padrão de análise utilizado no mapa conceitual 


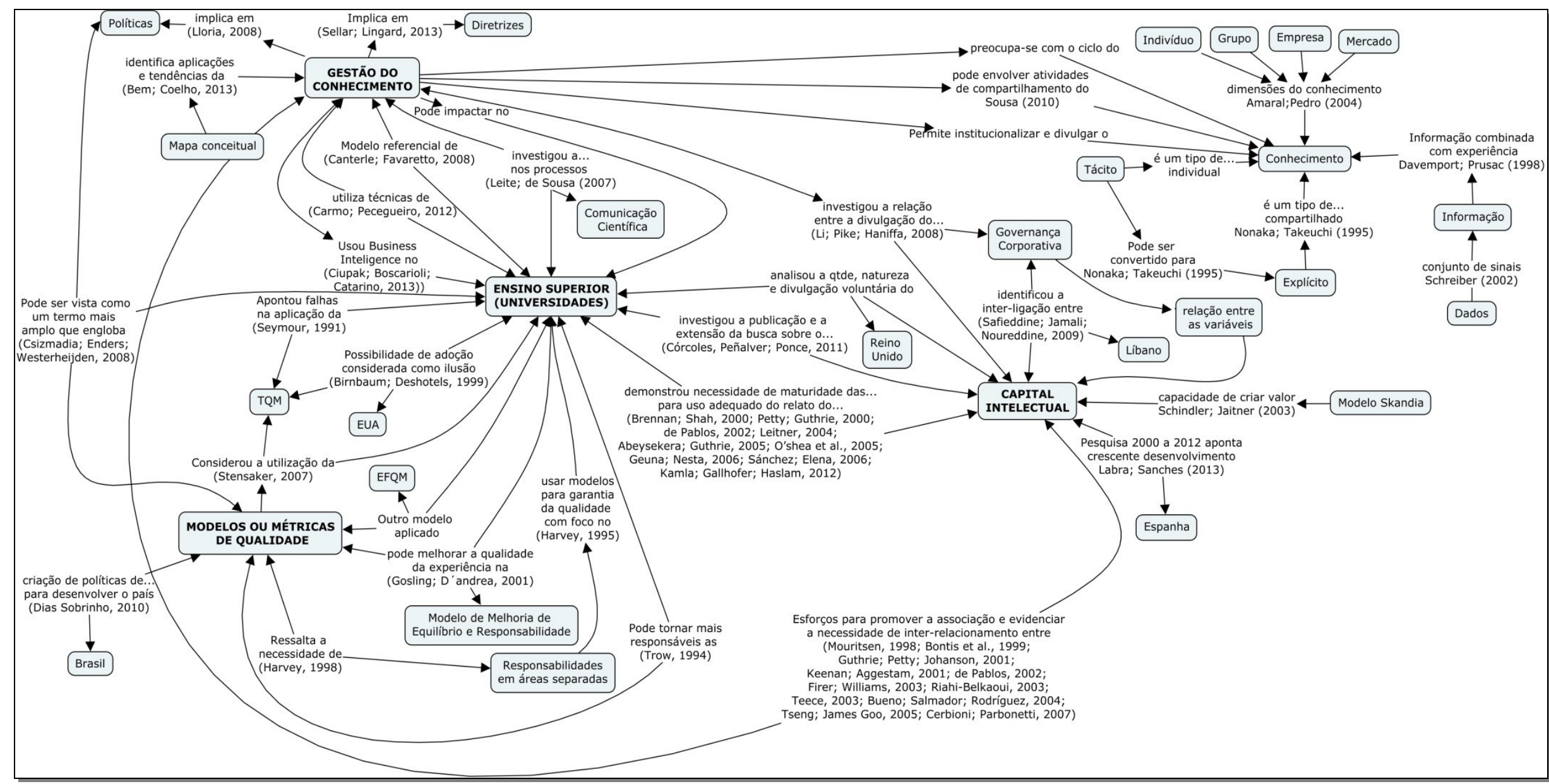

Figura 3 - Mapa Conceitual

Fonte: Elaborado de forma colaborativa pelos autores 
Os padrões de análise utilizados na construção do mapa conceitual são exemplificados na Figura 3, onde, por exemplo, é demonstrado o relacionamento entre as proposições dos autores: Birbaum e Deshotels (1999), Seymour (1991) e Stensaker (2007), que pode ser interpretada no mapa conceitual como: A possibilidade de adoção do TQM foi considerada como ilusão no ensino superior dos EUA (BIRBAUM; DESHOTELS, 1999). Seymour (1991) apontou falhas na aplicação da TQM no ensino superior e Stensaker (2007) considerou a utilização de métricas de qualidade na TQM.

Após a apresentação do mapa conceitual foi possível delinear o modelo conceitual da pesquisa como contribuição deste trabalho. O modelo conceitual é apresentado a seguir.

\subsection{MODELO CONCEITUAL DA REVISÃO DE LITERATURA}

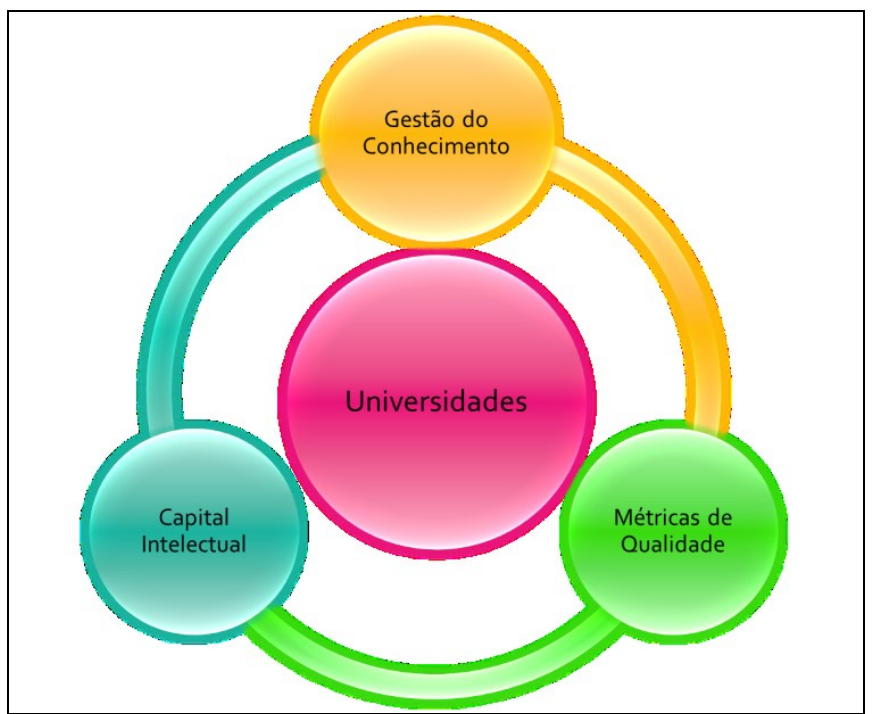

Figura 4 Modelo conceitual da revisão de literatura Fonte: Elaborado pelos autores

O modelo conceitual da investigação tem como objetivo evidenciar de qual forma ocorreu a inter-relação entre os conceitos de capital intelectual, gestão do conhecimento e a utilização de padrões para as métricas de qualidade cenário do ensino superior.

A Figura 4 demonstrou o modelo conceitual resultante da análise do mapa conceitual da revisão de literatura deste trabalho. O modelo expressa clara e diretamente a inter-relação entre as dimensões avaliadas no cenário do ensino superior, neste contexto representado pelas universidades e sintetiza os conceitos demonstrados no mapa conceitual, oriundos da revisão de literatura.

A próxima seção apresenta as conclusões do trabalho. 


\section{CONCLUSÃO}

Este trabalho apresentou uma revisão de literatura com o objetivo de compreender e identificar a existência de uma inter-relação entre a gestão do conhecimento, mensuração do capital intelectual e as diversas métricas de qualidade no ensino superior.

Ao considerar que o conhecimento é um dos mais importantes ativos no novo contexto das IES, verificou-se que a literatura quanto ao relato do capital intelectual ainda apresenta-se em estágio de desenvolvimento (KAMLA; GALLHOFER; HASLAM, 2012; LABRA; SÁNCHEZ, 2013).

O modelo conceitual resultante da revisão de literatura pode ser validado com base nos achados que indicaram a estreita interligação entre os conceitos apresentados no mapa conceitual. O conjunto da revisão de literatura, mapa conceitual e modelo conceitual podem colaborar para o enriquecimento e entendimento do tema trabalhado, permitindo, pela sua característica inovadora, atestar visualmente o hiato quanto à forma de abordagem.

A utilização de mapa conceitual para evidenciar os principais conceitos e relacionamentos oriundos da revisão de literatura permitiu organizar e inter-relacionar o conhecimento das áreas investigadas. Pode-se avaliar positivamente a utilização de mapas conceituais na elicitação do conhecimento oriundo da revisão de literatura, pois este recurso permitiu externalizar, de forma explícita, conceitos e ligações que poderiam passar despercebidos em uma análise textual isolada.

Embora a literatura que trata da divulgação do relato do CI ainda apresente-se em estágio de desenvolvimento e maturidade, o modelo conceitual resultante da revisão de literatura pode ser validado com base nos achados que indicaram a estreita interligação entre os conceitos apresentados no mapa conceitual.

As métricas de qualidade podem ser consideradas, no contexto educacional, como indutoras de boas práticas de governança corporativa e têm ganhado espaço no cenário da gestão do conhecimento e do capital intelectual.

Evidenciou-se que as métricas de qualidade têm sido amplamente desenvolvidas e utilizadas no cenário das universidades, e, no contexto da gestão contemporânea, podem ser visivelmente consideradas necessárias, principalmente considerando-se a exigência das universidades serem aprovadas no processo de avaliação e acreditação do ensino superior. 
A integração destas dimensões pode ser considerada viável e de cariz qualitativa quando utilizada adequadamente pelas universidades como forma de obter vantagem competitiva no contexto do ensino superior.

A utilização de mapa conceitual para apresentar os principais conceitos e relacionamentos oriundos da revisão de literatura permitiu organizar e inter-relacionar o conhecimento das áreas investigadas. Pode-se avaliar positivamente a utilização de mapas conceituais na elicitação do conhecimento oriundo da revisão de literatura, pois este recurso permitiu externalizar, de forma explícita, conceitos e ligações que poderiam passar despercebidos em uma análise textual isolada.

\section{REFERÊNCIAS}

ABEYSEKERA, I.; GUTHRIE, J. An empirical investigation of annual reporting trends of intellectual capital in Sri Lanka. Critical Perspectives on accounting, v. 16, n. 3, p. 151$163,2005$.

AMARAL, P. C.; PEDRO, J. M. O Capital Conhecimento: modelos de avaliação de activos intangíveis. Lisboa. Universidade Católica Portuguesa, 2004.

BEM, R. M. DE; COELHO, C. C. DE S. R. Aplicações da gestão do conhecimento na área de biblioteconomia e ciência da informação: uma revisão sistemática. Brazilian Journal of Information Science; Vol. 7, No 1 (2013), 2013.

BEZHANI, I. Intellectual capital reporting at UK universities. Journal of Intellectual Capital, v. 11, n. 2, p. 179-207, 2010.

BIRNBAUM, R.; DESHOTELS, J. Has the Academy Adopted TQM?. Planning for Higher Education, v. 28, n. 1, p. 29-37, 1999.

BONTIS, N. et al. The knowledge toolbox: A review of the tools available to measure and manage intangible resources. European management journal, v. 17, n. 4, p. 391-402, 1999. BRENNAN, J.; SHAH, T. Managing quality in higher education: An international perspective on institutional assessment and change. [s.1.] Organisation for economic cooperation and development Buckingham, 2000.

BUENO, E.; SALMADOR, M. P.; RODRÍGUEZ, Ó. The role of social capital in today's economy: empirical evidence and proposal of a new model of intellectual capital. Journal of Intellectual Capital, v. 5, n. 4, p. 556-574, 2004.

CAÑAS, A. J. et al. Herramientas para construir y compartir modelos de conocimiento basados en mapas conceptuales. Revista de Informática Educativa, v. 13, n. 2, p. 145-158, 2000 . 
CANTERLE, N. M. G.; FAVARETTO, F. A reference model proposal for management of the quality indicators at universities. Proposta de um modelo referencial de gestão de indicadores de qualidade na instituição universitária, v. 16, n. 60, p. 393-412, 2008.

CARMO, J. R. DO; PECEGUEIRO, C. M. P. DE A. Organização do conhecimento científico na universidade: um estudo de caso. Brazilian Journal of Information Science; Vol. 5, No 2 (2011), 2012.

CERBIONI, F.; PARBONETTI, A. Exploring the effects of corporate governance on intellectual capital disclosure: an analysis of European biotechnology companies. European Accounting Review, v. 16, n. 4, p. 791-826, 2007.

CIUPAK, L. F.; BOSCARIOLI, C.; CATARINO, M. E. Análise do uso de tecnologias de business intelligence como facilitadoras à gestão universitária. Brazilian Journal of Information Science; Vol. 7 (2013): Número Especial - 1. Sem., 2013.

CÓRCOLES, Y. R.; PEÑALVER, J. F. S.; PONCE, Á. T. Beneficios y costes de la divulgación de información sobre capital intelectual: Evidencia empírica en las universidades públicas españolas. Estudios de Economía Aplicada, v. 50, p. 23-29, 2011.

CSIZMADIA, T.; ENDERS, J.; WESTERHEIJDEN, D. F. Quality management in Hungarian higher education: Organisational responses to governmental policy. Higher Education, v. 56, n. 4, p. 439-455, 2008.

DAVENPORT, T. H.; PRUSAK, L. Working Knowledge: How Organizations Manage

What They Know. Cambridge, Massachusetts: Harvard Business School Press., 1998.

DE PABLOS, P. O. Evidence of intellectual capital measurement from Asia, Europe and the Middle East. Journal of Intellectual Capital, v. 3, n. 3, p. 287-302, 2002.

DEAN, G.; GOTTSCHALK, P. Knowledge management in policing and law enforcement: foundations, structures and applications. Oxford: Oxford University Press, 2007.

DIAS SOBRINHO, J. Avaliação e transformações da educação superior brasileira (19952009): do provão ao Sinaes. Avaliação: Revista da Avaliação da Educação Superior (Campinas), v. 15, p. 195-224, 2010.

EDVINSSON, L.; MALONE, M. S. Intellectual Capital: Realizing Your Company’s True Value by Finding its Hidden Brainpower. 1997.

FIRER, S.; WILLIAMS, S. M. Intellectual capital and traditional measures of corporate performance. Journal of Intellectual Capital, v. 4, n. 3, p. 348-360, 2003.

GEUNA, A.; NESTA, L. J. J. University patenting and its effects on academic research: The emerging European evidence. Research Policy, v. 35, n. 6, p. 790-807, 2006.

GOSLING, D.; D’ANDREA, V.-M. Quality development: a new concept for higher education. Quality in higher education, v. 7, n. 1, p. 7-17, 2001. 
GUTHRIE, J.; PETTY, R.; JOHANSON, U. Sunrise in the knowledge economy: managing, measuring and reporting intellectual capital. Accounting, Auditing \& Accountability Journal, v. 14, n. 4, p. 365-384, 2001.

HAGEMANS, M. G.; VAN DER MEIJ, H.; DE JONG, T. The effects of a concept mapbased support tool on simulation-based inquiry learning.Journal of Educational PsychologyHagemans, Mieke G.: Department of Instructional Technology, University of Twente, PO Box 217, Enschede, Netherlands, 7500 AE, m.g.hagemans@utwente.nlAmerican Psychological Association, , 2013.

HARVEY, L. Beyond tqm. Quality in Higher Education, v. 1, n. 2, p. 123-146, 1995. An assessment of past and current approaches to quality in higher education. Australian Journal of Education, v. 42, n. 3, p. 237-255, 1998.

KAMLA, R.; GALLHOFER, S.; HASLAM, J. Understanding Syrian accountants' perceptions of, and attitudes towards, social accounting. Accounting, Auditing and Accountability Journal, v. 25, n. 7, p. 1170-1205, 2012.

KEENAN, J.; AGGESTAM, M. Corporate governance and intellectual capital: some conceptualisations. Corporate Governance: An International Review, v. 9, n. 4, p. 259$275,2001$.

KELLY, A. The intellectual capital of schools: analysing government policy statements on school improvement in light of a new theorization. Journal of Education Policy, v. 19, n. 5, p. 609-629, 1 set. 2004.

LABRA, R.; SÁNCHEZ, M. P. National intellectual capital assessment models: a literature review. Journal of Intellectual Capital, v. 14, n. 4, p. 582-607, 2013.

LEITE, F. C. L.; DE SOUZA COSTA, S. M. Scientific knowledge management: Proposal for a conceptual model based on processes of scientific communication. Gestão do conhecimento científico: Proposta de um modelo conceitual com base em processos de comunicação científica, v. 36, n. 1, p. 92-107, 2007.

LEITNER, K.-H. Intellectual capital reporting for universities: conceptual background and application for Austrian universities. Research Evaluation, v. 13, n. 2, p. 129-140, 2004.

LI, J.; PIKE, R.; HANIFFA, R. Intellectual capital disclosure and corporate governance structure in UK firms. Accounting and Business Research, v. 38, n. 2, p. 137-159, 2008.

LLORIA, M. B. A review of the main approaches to knowledge management. Knowledge Management Research \& Practice, v. 6, n. 1, p. 77-89, 2008.

MENDELEY, S. T. Mendeley Desktop. version 1.10.1: Reference Manager Mendeley Desktop. Mendeley Ltd., , 2013. Disponível em: <http:/www.mendeley.com> 
MOON, B. et al. Applied concept mapping: Capturing, analyzing, and organizing knowledge. Boca Ratom. Flórida: CRC Press Taylor \& Francis Group, 2011.

MOURITSEN, J. Driving growth: economic value added versus intellectual capital. Management accounting research, v. 9, n. 4, p. 461-482, 1998.

NONAKA, I.; TAKEUCHI, H. The knowledge-creating company: How Japanese companies create the dynamics of innovation. Long range planning, v. 29, n. 4, p. 592, 1995.

O'SHEA, R. P. et al. Entrepreneurial orientation, technology transfer and spinoff performance of US universities. Research Policy, v. 34, n. 7, p. 994-1009, 2005.

PETTY, R.; GUTHRIE, J. Intellectual capital literature review: measurement, reporting and management. Journal of intellectual capital, v. 1, n. 2, p. 155-176, 2000.

RIAHI-BELKAOUI, A. Intellectual capital and firm performance of US multinational firms: a study of the resource-based and stakeholder views. Journal of Intellectual capital, v. 4, n. 2, p. 215-226, 2003.

SAFIEDDINE, A.; JAMALI, D.; NOUREDDINE, S. Corporate governance and intellectual capital: evidence from an academic institution. Corporate Governance, v. 9, n. 2, p. 146$157,2009$.

SÁNCHEZ, M. P.; ELENA, S. Intellectual capital in universities: Improving transparency and internal management. Journal of Intellectual Capital, v. 7, n. 4, p. 529-548, 2006.

SCHINDLER, R.; JAITNER, A. Intellectual capital: measuring knowledge management. In: Knowledge Management. Germany: Springer, 2003. p. 151-175.

SCHREIBER, G. ET AL. Knowledge engnineering and management: the CommonKADS methodology. Cambridge/Massachussets: MIT Press, 2002. p. 932

SELLAR, S.; LINGARD, B. The OECD and global governance in education. Journal of Education Policy, v. 28, n. 5, p. 710-725, 2013.

SEYMOUR, D. T. TQM on Campus: What the Pioneers Are Finding. AAHE Bulletin, v. 44, n. 3, p. 10-13, 1991.

On Q: Causing Quality in Higher Education. 100 Front St., Riverside, NJ: Macmillan Publishing Company/ERIC, 1992.

SILVA, A. C. R. DA. Metodologia da Pesquisa Aplicada à Contabilidade. 2 ed. ed. São Paulo: Atlas Editora., 2008.

SOUZA, L. L. C. Práticas da gestão do conhecimento na cadeia de valor terceirizada: Estudo no Setor Elétrico. [s.1.] Tese de Doutorado. Universidade Federal de Santa Catarina. Florianópolis/Santa Catarina, 2010. 
STENSAKER, B. Quality as fashion: exploring the translation of a management idea into higher education. In: Quality assurance in higher education. [s.1.] Springer, 2007. p. 99118.

TEECE, D. J. Capturing value from knowledge assets: the new economy, markets for knowhow and intangible assets. Essays on Technology Management and Policy, p. 47-75, 2003.

TIWANA, A. The Knowledge Management toolkit. 2. ed. Canada: Prentice Hall, 2002.

TROW, M. Academic reviews and the culture of excellence. [s.1.] Universitetskanslern, Kanslersämbetet, 1994.

TSENG, C.; JAMES GOO, Y. Intellectual capital and corporate value in an emerging economy: empirical study of Taiwanese manufacturers. R\&D Management, v. 35, n. 2, p. 187-201, 2005. 